\title{
Effects of sea squirt (Halocynthia roretzi) lipids on white adipose tissue weight and blood glucose in diabetic/obese $\mathrm{KK}-A^{y}$ mice
}

\author{
NANA MIKAMI, MASASHI HOSOKAWA and KAZUO MIYASHITA \\ Faculty of Fisheries Sciences, Hokkaido University, Hokkaido 041-8611, Japan
}

Received January 5, 2010; Accepted February 22, 2010

DOI: 10.3892/mmr_00000278

\begin{abstract}
Lipids extracted from Halocynthia roretzi contain n-3 polyunsaturated fatty acids such as eicosapentaenoic acid and docosahexaenoic acid, as well as carotenoids. The aim of the present study was to evaluate the effect of $H$. roretz $i$ lipids on white adipose tissue (WAT) weight and high blood glucose levels in diabetic/obese $\mathrm{KK}-A^{y}$ mice. $H$. roretzi lipids were fed to the diabetic/obese $\mathrm{KK}-A^{y}$ mice for 5 weeks. In the mice treated with the $H$. roretzi lipids compared to control mice, WAT weight was reduced, blood glucose levels and leptin mRNA expression in the epididymal WAT were significantly decreased, serum leptin levels also tended to decrease, and serum adiponectin levels tended to increase. These results demonstrate that $H$. roretzi lipids have beneficial health effects on diabetic/obese KK- $A^{y}$ mice.
\end{abstract}

\section{Introduction}

Halocynthia roretzi (Maboya) is an edible marine invertebrate sea squirt with an orange-colored mantle covered by a hard tunic that is eaten in Japan and Korea. H. roretzi contains various functional components such as eicosapentaenoic acid (EPA), docosahexaenoic acid (DHA), carotenoids, taurine and plasmalogen (1-3). In a previous study, Kawasaki reported that hexane extracts from $H$. roretzi muscle decreased total cholesterol, triglycerides, phospholipids and NEFA in the serum of rats (4). We also reported that acethylene carotenoids such as alloxanthin and diatoxanthin contained in $H$. roretzi lipids suppress the secretion of proinflammatory cytokines such as interleukin- 6 and interleukin-1 $\beta$ from macrophage-like RAW 264.7 cells stimulated by lipopolysaccharides (5). In addition, halocynthiaxanthin and fucoxanthinol isolated from $H$. roretzi have been reported to induce apoptosis in human leukemia and colon cancer cells (6).

Correspondence to: Dr Masashi Hosokawa, Faculty of Fisheries Sciences, Hokkaido University, 3-1-1 Minato-cho, Hakodate, Hokkaido 041-8611, Japan

E-mail: hoso@fish.hokudai.ac.jp

Key words: Halocynthia roretzi, type 2 diabetes, hyperglycemia, blood glucose level, serum adipocytokine secretion
In recent years, the incidence of type 2 diabetes has increased worldwide. Diabetes, a state of high blood glucose level (also known as hyperglycemia), is closely associated with obesity. In hypertrophic adipose tissue in which excessive fat accumulation is observed in obesity, dysregulation of adipocytokine secretion occurs and induces insulin resistance (7). Therefore, it is crucial to prevent type 2 diabetes by ameliorating excessive fat accumulation and adipocytokine production in white adipose tissue (WAT).

In this study, we examined the effects of $H$. roretzi lipids on WAT weight and high blood glucose levels in diabetic/ obese KK- $A^{y}$ mice.

\section{Materials and methods}

Materials. H. roretzi was obtained from Kamiiso Fisheries Cooperative Association (Hokkaido, Japan). Sucrose and L-cystine used in animal diets were purchased from Kanto Chemical Co., Inc. (Tokyo, Japan). Choline bitartrate and tetra-butylhydroquinone were purchased from Sigma-Aldrich ${ }^{\circledR}$ (St. Louis, MO, USA). Soybean oil was purchased from Wako Pure Chemical Industries, Ltd. (Osaka, Japan). Other ingredients for the animal diets were obtained from Clea Japan, Inc. (Tokyo, Japan).

Extraction of $H$. roretzi lipids. The entire body of $H$. roretzi including the tunic was cut into small pieces, and crude lipids were extracted with ethanol for 4 days at room temperature under shade. The extraction was repeated twice. The pooled extracts were concentrated by evaporation, and water soluble components were removed from crude lipids using chloroformmethanol-water (10/5/3, v/v/v). H. roretzi lipids were obtained from the chloroform layer.

Animals. This animal experiment was approved by the Ethics Committee of Experimental Animal Care of Hokkaido University. KK- $A^{y}$ diabetic/obese mice (4-week-old, male) were obtained from Clea Japan, Inc. The mice $(n=6)$ were individually housed at $23 \pm 1^{\circ} \mathrm{C}$ and $45-60 \%$ humidity under a 12-h light/dark cycle. A diet prescribed by the American Institute of Nutrition (AIN-93G) (8) was used as the control diet. The composition of the control diet consisted of $10.0 \%$ soybean oil, $37.4 \%$ cornstarch, $12.4 \%$ dextrised cornstarch, $20.7 \%$ casein, $9.4 \%$ sucrose, $5.0 \%$ cellulose, $3.5 \%$ AIN-93G mineral mix, $1.0 \%$ AIN-93G vitamin mix, $0.3 \%$ L-cystine, 
$0.25 \%$ choline bitartrate and $0.0014 \%$ tetra-butylhydroquinone. After a 1-week acclimatization period with the control diet, the mice were randomly divided into two groups. The experimental group was fed the experimental diet containing $5 \%$ soybean oil $+5 \% H$. roretzi lipids $(5 \% H$. roretzi lipid diet). After 5 weeks of feeding, the mice were fasted for $12 \mathrm{~h}$, and blood was collected under anesthesia using diethyl ether. The liver and WAT were immediately excised and weighed. Parts of the epididymal and mesenteric WAT were conserved in RNAlater ${ }^{\circledR}$ (Sigma-Aldrich) to measure the adipocytokine mRNA expression level. The serum was separated from blood and stored at $-20^{\circ} \mathrm{C}$ for measurements of insulin, adiponectin and leptin levels.

Analysis of blood glucose levels. Blood glucose levels were measured using a blood glucose monitor (Glutest Neo Sensor; Sanwa Kagaku Kenkyusho Co., Ltd., Nagoya, Japan) in mice fed for 33 days with the control diet or the $5 \%$ H. roretzi lipid diet after $12 \mathrm{~h}$ of fasting. This sensor is based on the glucose dehydrogenase method.

Glucose tolerance test. After 27 days of feeding, a glucose tolerance test was performed. Mice were fasted for $4 \mathrm{~h}$, and D-glucose solution was administered ( $2 \mathrm{mg} / \mathrm{g}$ of body weight). Blood glucose levels were measured at 0, 15, 30, 60, 90, 120 and $150 \mathrm{~min}$ after glucose administration by a Glutest Neo Sensor.

Measurement of adipocytokine gene expression. Total RNA was extracted from RNAlater-treated epididymal and mesenteric WAT samples (<100 mg) using an RNeasy Lipid Tissue Mini kit (Qiagen, Tokyo, Japan) according to the manufacturer's protocol. cDNA was synthesized from total RNA by reverse transcription reactions using the HighCapacity cDNA Archive kit (Applied Biosystems Japan Ltd., Tokyo, Japan). Real-time quantitative PCR analysis was performed with an automated sequence detection system (ABI PRISM 7500; Applied Biosystems Japan Ltd.). The cycling conditions of PCR were $50^{\circ} \mathrm{C}$ for $2 \mathrm{~min}$ and $95^{\circ} \mathrm{C}$ for $10 \mathrm{~min}$, followed by 40 cycles at $95^{\circ} \mathrm{C}$ for $15 \mathrm{sec}$ and $60^{\circ} \mathrm{C}$ for $1 \mathrm{~min}$. Adiponectin, leptin and glyceraldehyde-3-phosphate dehydrogenase (GAPDH) mRNA expression levels were measured by Taq Man Gene Expression assays from Applied Biosystems Japan Ltd. PCR primers (adiponectin, Mm00456425_m1; leptin, Mm00434759_m1; GAPDH, Mm99999915_g1) were also purchased from Applied Biosystems Japan Ltd.

Measurement of insulin, adiponectin and leptin concentrations in serum. Serum insulin, leptin and adiponectin levels were measured using the mouse insulin ELISA kit (H-type), the mouse leptin ELISA kit (Shibayagi Co., Ltd., Gunma, Japan) and the mouse/rat adiponectin ELISA kit (Otsuka Pharmaceutical Co., Ltd., Tokyo, Japan), respectively, according to the manufacturer's protocol.

Statistical analysis. Data were expressed as the mean \pm standard error (SE). Statistical significance was determined between the two groups using Welch's t-test. A significant difference was defined at $\mathrm{P}<0.05$ or $\mathrm{P}<0.01$.
Table I. Effect of $H$. roretzi lipids on the growth parameters of $\mathrm{KK}-A^{y}$ mice.

\begin{tabular}{lrc}
\hline & Control & H. roretzi lipids \\
\hline Final body weight $(\mathrm{g})$ & $37.3 \pm 1.2$ & $38.8 \pm 1.0$ \\
Change in body weight $(\mathrm{g})$ & $10.3 \pm 0.8$ & $11.4 \pm 0.7$ \\
Food intake (g/day) & $3.9 \pm 0.4$ & $4.4 \pm 0.2$ \\
\hline
\end{tabular}

Values are expressed as the mean $\pm \operatorname{SE}(n=6)$.

\section{Results and Discussion}

The yield of lipids obtained from the entire body of $H$. roretzi was $5.1 \%$ yield per dry weight. The lipid composition was 49.1\% neutral lipids, $38.4 \%$ polar lipids containing carotenoids and $12.5 \%$ others. The fatty acid composition of $H$. roretzi lipids was mainly $11.4 \%$ palmitic acid, $27.2 \%$ EPA and $13.8 \%$ DHA, respectively. In addition, alloxanthin, halocynthiaxanthin and diatoxanthin were detected in $H$. roretz $i$ lipids using high performance liquid chromatography as previously reported $(2,5)$.

To investigate the effects of $H$. roretzi lipids on WAT weight and blood glucose level, $\mathrm{KK}-A^{y}$ diabetic/obese mice were fed a diet containing $5 \%$ H. roretzi lipids $+5 \%$ soybean oil for 5 weeks. Control mice were fed a diet containing $10 \%$ soybean oil. No significant difference in food intake and body weight gain was noticed between the two groups during the experimental period (Table I). However, the WAT weight to body weight was significantly lower in the mice fed the diet containing $5 \% \mathrm{H}$. roretzi lipids compared to the control mice (Fig. 1). In addition, the $5 \% \mathrm{H}$. roretzi lipid diet significantly decreased serum LDL-cholesterol levels compared to the control (Fig. 2). Triglyceride, free fatty acids and total cholesterol levels did not differ between the two groups. Kawasaki reported that hexane extracts from $H$. roretzi decreased VLDL and LDL-cholesterol in Wistar rats (4). H. roretzi lipids extracted with ethanol had the same effect on the LDL-cholesterol of the diabetic/obese KK- $A^{y}$ mice.

Blood glucose levels were also significantly lower in the mice fed the diet containing $5 \% H$. roretzi lipids compared to the control mice after 33 days of feeding and $12 \mathrm{~h}$ of fasting (Fig. 3). Furthermore, serum insulin levels in mice fed the diet containing $5 \% \mathrm{H}$. roretzi lipids tended to decrease compared to the control mice (Fig. 3). To evaluate the insulin sensitivity of the KK- $A^{y}$ mice fed the diet containing $5 \% H$. roretzi lipids, we performed a glucose tolerance test. After administration of glucose $(2 \mathrm{mg} / \mathrm{g}$ of body weight), the blood glucose concentrations of the mice fed the diet containing $5 \% \mathrm{H}$. roretz $i$ lipids were significantly low at all time intervals as compared to those of the control mice, although the reduction in the blood glucose levels of the $5 \% \mathrm{H}$. roretzi lipid-fed mice was not significantly different from that of the control mice (Fig. 4). $\mathrm{KK}-A^{y}$ mice are known to have hyperglycemia, hyperinsulinemia and hyperleptinemia, and exhibit insulin resistance along with type 2 diabetes. Our results suggest that $H$. roretzi lipids are effective for preventing or improving hyperglycemia through the attenuation of WAT weight gain in $\mathrm{KK}-A^{y}$ 
Liver

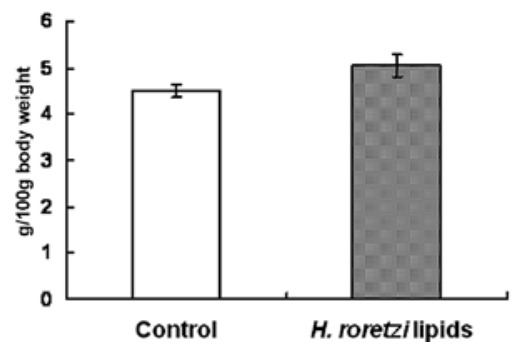

White adipose tissue

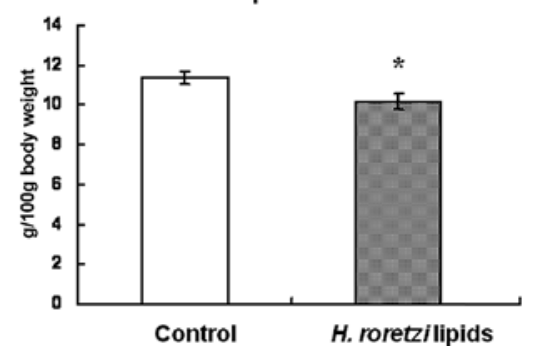

Figure 1. Effect of H. roretzi lipids on liver and white adipose tissue weight in KK-Ay mice. Mice were fed the control or $5 \%$ H. roretzi lipid diets for 5 weeks. WAT weight: total weight of epididymal, mesenteric, perirenal, retroperitoneal and inguinal white adipose tissues. Values are expressed as mean $\pm \mathrm{SE}(\mathrm{n}=6)$. ${ }^{*} \mathrm{P}<0.05$ vs. control.
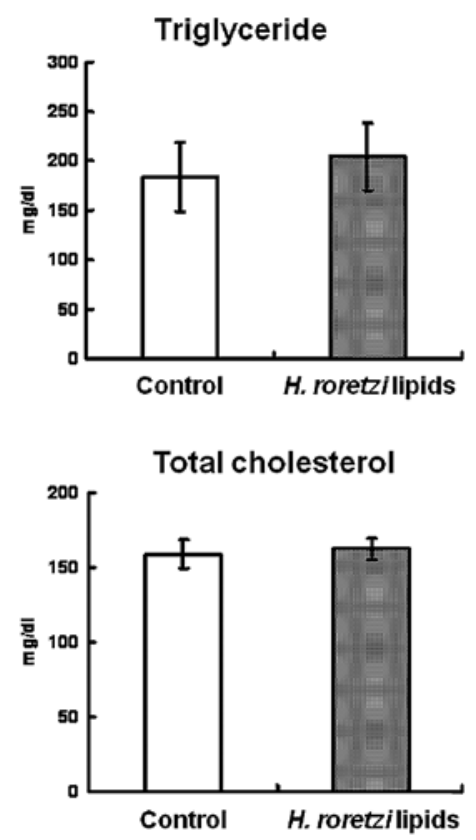
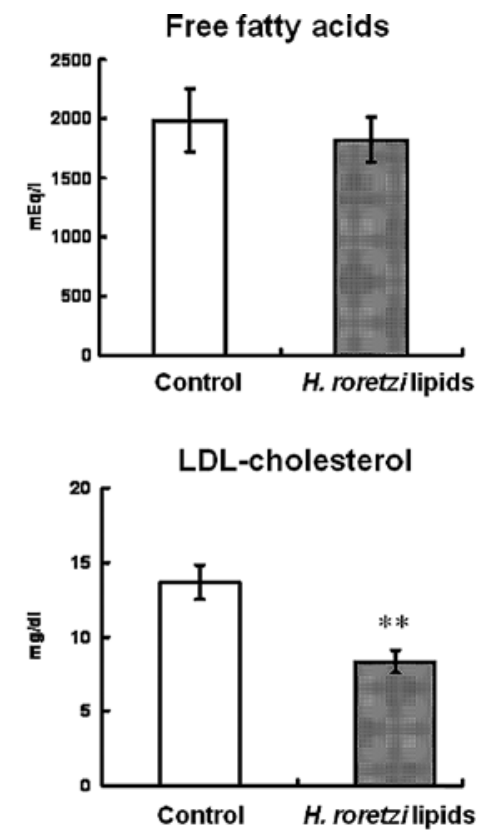

Figure 2. Effect of $H$. roretzi lipids on serum lipid parameters in KK- $A^{y}$ mice. The mice were fed the control or $5 \%$ H. roretzi lipid diet for 5 weeks. Serum triglyceride, free fatty acids, total cholesterol and LDL-cholesterol levels were measured after $12 \mathrm{~h}$ of fasting. Values are expressed as the mean $\pm \mathrm{SE}(\mathrm{n}=6)$. ${ }^{* *} \mathrm{P}<0.01$ vs. control.

Blood glucose (33 days)

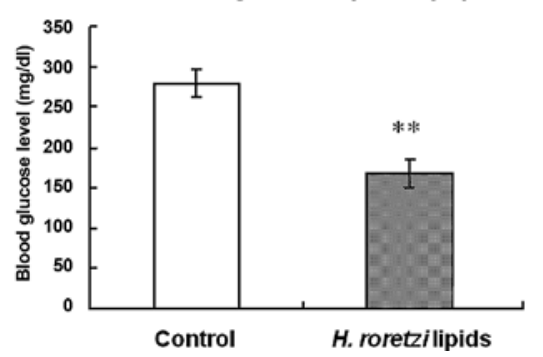

Serum insulin (5 weeks)

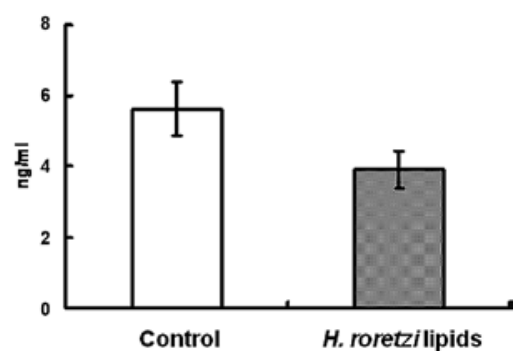

Figure 3. Effect of $H$. roretzi lipids on blood glucose and serum insulin levels in KK- $A^{y}$ mice. Mice were fed the control or $5 \%$. roretzi lipid diet for 5 weeks. Blood glucose levels were measured after $12 \mathrm{~h}$ of fasting in mice which had been fed the diets for 33 days. Furthermore, the serum insulin level was measured by ELISA after $12 \mathrm{~h}$ of fasting in mice fed the diets for 5 weeks. Values are expressed as the mean $\pm \mathrm{SE}(\mathrm{n}=6)$. ${ }^{* *} \mathrm{P}<0.01 \mathrm{vs}$. control.

mice. Adiponectin up-regulates insulin signaling by activating PPAR $\alpha$ and AMP kinase (9). In hypertrophied adipose tissue observed in obese mice, dysregulation of adipocytokine secretion occurs and induces insulin sensitivity. Adiponectin, in particular, is known to be reduced in obese states (10). In this study, the serum adiponectin and adiponectin mRNA expression levels in mesenteric WAT in the $5 \%$ H. roretzi lipid diet-fed mice tended to increase, although not significantly, as compared to the control mice (Figs. 5 and 6A). In addition, serum leptin levels tended to decrease and leptin mRNA 


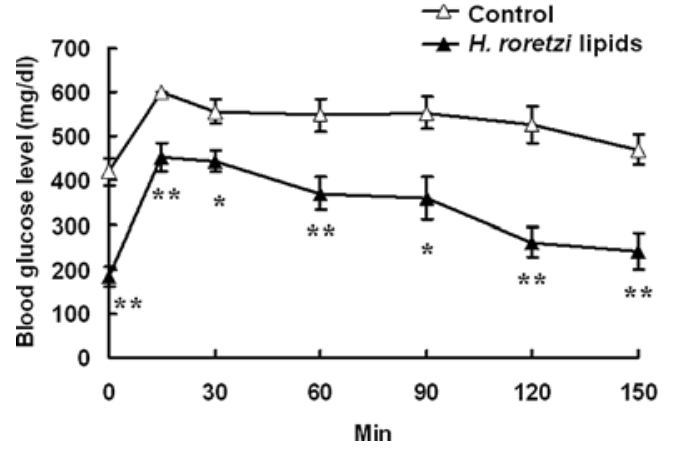

Figure 4. Glucose tolerance test in KK- $A^{y}$ mice fed a diet containing $H$. roretzi lipids. Mice were fasted for $4 \mathrm{~h}$ after 4 weeks of feeding and were then orally administered D-glucose solution ( $2 \mathrm{mg} / \mathrm{g}$ of body weight). After $0,15,30,60$, 90,120 and 150 min of administration, the blood glucose levels were measured using the Glutest Neo Sensor. Blood glucose levels $>600 \mathrm{mg} / \mathrm{dl}$ were calculated as $600 \mathrm{mg} / \mathrm{dl}$, which was the maximum value of the Glutest Neo Sensor. Values are expressed as the mean $\pm \mathrm{SE}(\mathrm{n}=6)$. ${ }^{*} \mathrm{P}<0.05$ vs. control. ${ }^{* *} \mathrm{P}<0.01$ vs. control. expression levels in epididymal WAT were significantly lower in mice fed the diet containing $5 \% \mathrm{H}$. roretzi lipids than in the control mice (Figs. 5 and 6B). Serum leptin is known to be correlated positively to fat mass (11). Therefore, the results in the $5 \% \mathrm{H}$. roretzi lipid group suggest that the serum leptin levels were dependent on the suppression of WAT accumulation in the KK- $A^{y}$ mice.

The fatty acid composition of $H$. roretzi lipids was $27.2 \%$ EPA and $13.8 \%$ DHA. These n-3 polyunsaturated fatty acids have been reported to suppress fat accumulation in WAT and to decrease high blood glucose levels in genetically induced diabetic/obese mice (12) and high fat-induced obese mice (13). However, the EPA and DHA concentrations in the 5\% $H$. roretzi lipid diet were 0.48 and $0.25 \%$, which is lower than in previous reports $(12,13)$. In addition, we did not observe a suppressive effect on WAT weight gain and blood glucose levels in KK- $A^{y}$ mice fed a diet containing $7 \%$ fish
Serum adiponectin

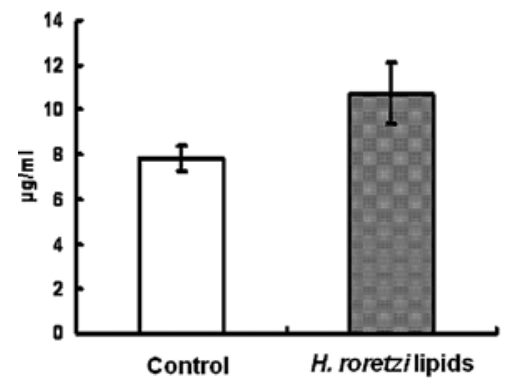

Serum leptin

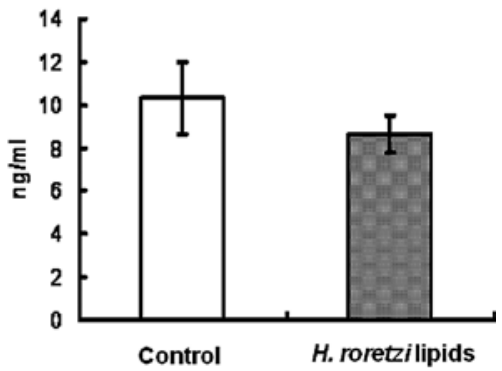

Figure 5. Effects of $H$. roretzi lipids on serum adiponectin and leptin levels in KK- $A^{y}$ mice after 5 weeks of feeding with the control or $5 \%$. roretzi lipid diet. Blood samples were collected after $12 \mathrm{~h}$ of fasting, and adipocytokine levels were measured by ELISA. Values are expressed as the mean \pm SE $(\mathrm{n}=6$ ).

A

\section{Mesenteric WAT}

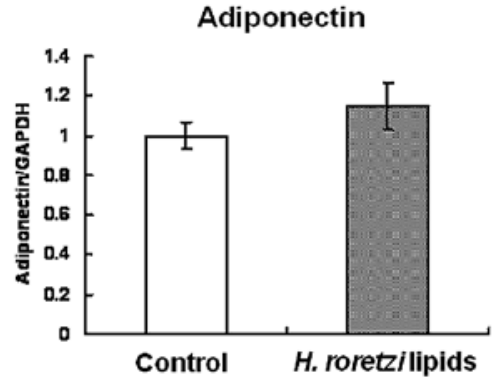

\section{B Epididymal WAT}

Adiponectin

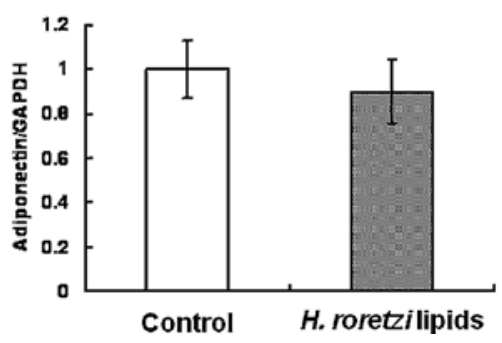

Leptin

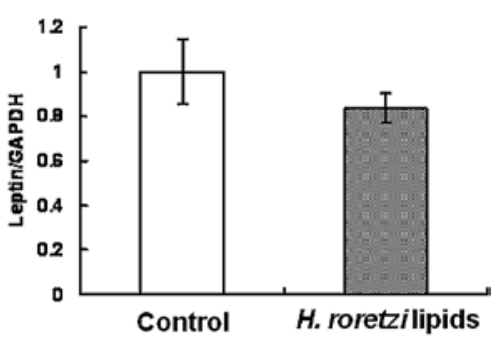

Leptin

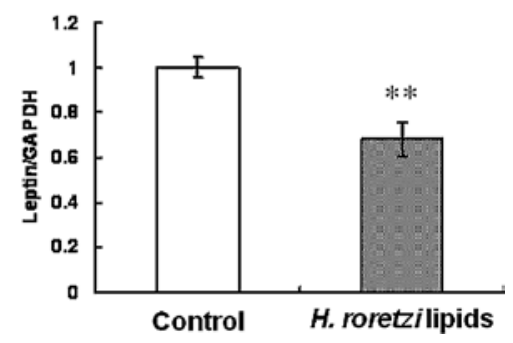

Figure 6. Effects of $H$. roretzi lipids on adiponectin and leptin mRNA expression levels in the mesenteric (A) and epididymal (B) WAT of KK- $A^{y}$ mice after 5 weeks of feeding with the control or $5 \%$ H. roretzi lipid diet. Values are expressed as the mean \pm SE $(n=6) .{ }^{* *} \mathrm{P}<0.01$ vs. control. 
oil (14). Therefore, the suppressive effects of $5 \% \mathrm{H}$. roretzi lipids on WAT weight gain and hyperglycemia in the $\mathrm{KK}-A^{y}$ mice depended not only on EPA and DHA, but also on other compounds. Furthermore, it is possible that n-3 PUFAs and other compounds had a combined effect. Thus, further investigation is required to clarify the mechanisms of $H$. roretzi lipids.

In conclusion, the present study indicates that $H$. roretzi lipids suppress fat accumulation in WAT and reduce high blood glucose levels in diabetic/obese $\mathrm{KK}-A^{y}$ mice. Furthermore, a decrease in serum LDL-cholesterol level was observed in $\mathrm{KK}-A^{y}$ mice fed a diet containing $5 \% H$. roretz $i$ lipids.

\section{References}

1. Jeong BY, Ohshima T and Koizumi C: Hydrocarbon chain distribution of ether phospholipids of the ascidian Halocynthia roretzi and the sea urchin Strongylocentrotus intermedius. Lipids 31: 9-18, 1996.

2. Matsuno T, Ookubo M, Nishizawa T and Shimizu I: Carotenoids of sea squirts. I. New marine carotenoids, halocynthiaxanthin and mytiloxanthinone from Halocynthia roretzi. Chem Pharm Bull 32: 4309-4315, 1984

3. Watanabe K, Uehara H, Sato M and Konosu S: Seasonal variation of extractive nitrogeneous constituents in the muscle of ascidian Halocynthia roretzi. Bull Japan Soc Sci Fish 51: 1293-1298, 1985.

4. Kawasaki M: Effects of dietary sea squirt (Halocynthia roretzi) on lipid metabolism in rats. Biofactors 22: 169-172, 2004.

5. Konishi I, Hosokawa M, Sashima T, Maoka T and Miyashita K Suppressive effects of alloxanthin and diatoxanthin from Halocynthia roretzi on LPS-induced expression of pro-inflammatory genes in RAW264.7 cells. J Oleo Sci 57: 181-189, 2008.
6. Konishi I, Hosokawa M, Sashima T, Kobayashi H and Miyashita K: Halocynthiaxanthin and fucoxanthinol isolated from Halocynthia roretzi induce apoptosis in human leukemia, breast and colon cancer cells. Comp Biochem Physiol C 142: 53-59, 2006.

7. Schäffler A, Schölmerich J and Büchler C: Mechanisms of disease: adipocytokines and visceral adipose tissue - emerging role in nonalcoholic fatty liver disease. Nat Clin Pract Gastroenterol Hepatol 2: 273-280, 2005.

8. Reeves PG, Nielsen, FH and Fahey GC: AIN-93 purified diets for laboratory rodents final report of the American Institute of Nutrition ad hoc writing committee on the reformulation of the AIN-76A rodent diet. J Nutr 123: 1939-1951, 1993.

9. Kadowaki $\mathrm{T}$ and Yamauchi $\mathrm{T}$ : Adiponectin and adiponectin receptors. Endocr Rev 26: 439-451, 2005.

10. Kadowaki T, Yamauchi T, Kubota N, Hara K, Ueki K and Tobe K: Adiponectin and adiponectin receptors in insulin resistance, diabetes, and the metabolic syndrome. J Clin Invest 116: 1784-1792, 2006

11. Sjögren K, Hellberg N, Bohlooly-YM, Savendahl L, Johansson MS, Berglindh T, Bosaeus I and Ohlsson C: Body fat content can be predicted in vivo in mice using a modified dualenergy X-ray absorptiometry technique. J Nutr 131: 2963-2966, 2001.

12. Hun CS, Hasegawa K, Kawabata T, Kato M, Shimokawa T and Kagawa Y: Increased uncoupling protein 2 mRNA in white adipose tissue, and decrease in leptin, visceral fat, blood glucose and cholesterol in $\mathrm{KK}-\mathrm{A}^{\mathrm{y}}$ mice fed with eicosapentaenoic and docosahexaenoic acids in addition to linolenic acid. Biochem Biophys Res Commun 259: 85-90, 1999.

13. Ikemoto S, Takahashi M, Tsunoda N, Maruyama K, Itakura $\mathrm{H}$ and Ezaki O: High-fat diet-induced hyperglycemia and obesity in mice: differential effects of dietary oils. Metabolism 5: 1539-1546, 1996

14. Maeda H, Hosokawa M, Sashima T and Miyashita K: Dietary combination of fucoxanthin and fish oil attenuates the weight gain of white adipose tissue and decreases blood glucose in obese/diabetic KK-Ay mice. J Agric Food Chem 55: 7701-7706, 2007. 